\title{
A PROOF OF A CONJECTURE OF A. H. STONE
}

\author{
R. F. DICKMAN, JR. ${ }^{1}$
}

\begin{abstract}
In this paper we use the techniques of analytic topology to establish a conjecture of A. H. Stone: A perfectly normal, locally connected, connected space is multicoherent if and only if there exist four nonempty, closed and connected subsets $A_{0}, A_{1}, A_{2}, A_{3}$ of $X$ such that $\bigcup_{i=0}^{3} A_{i}=X$ and the nerve of $\left\{A_{0}, A_{1}, A_{2}, A_{3}\right\}$ forms a closed 4-gon, i.e. $A_{i}$ meets $A_{i+1}$ and $A_{i-1}$ and no others (the suffices being taken mod 4).
\end{abstract}

1. Introduction. Throughout this paper $X$ will denote a locally connected, connected normal space. A region in $X$ is an open connected subset of $X$ and a continuum in $X$ is a closed (not necessarily compact) connected subset of $X$. We say that $X$ is unicoherent provided, whenever $X=H \cup K$ where $H$ and $K$ are continua, $H \cap K$ is connected and we say that $X$ is multicoherent if $X$ is not unicoherent.

In 1971, the author wrote A. H. Stone asking for his opinion concerning the following conjecture:

$X$ is unicoherent if and only if every pair of disjoint nonempty continua in $X$ can be separated by a continuum in $X$.

Professor Stone replied that $(*)$ seemed to be a special case $(n=4)$ of one of his conjectures. Let $n>2$ be an integer. We define the conjecture $S(n)$ by:

$S(n) \equiv X$ is multicoherent if and only if $X$ can be represented as the union of $n$ closed and connected continua, $\left\{A_{0}, A_{1}, \ldots, A_{n-1}\right\}$ whose nerve is a closed $n$-gon, i.e. $A_{i}$ meets $A_{i-1}$ and $A_{i+1}$ and no others (the suffixes being taken $\bmod n$ ).

Stone announced $S(3)$ in [7] and stated (in a private communication) that he was able to establish $S(n)$ for all $n>2$ only under every strong hypotheses, such as $X$ as finitely multicoherent (see [3] for definition).

In [5], it was shown that (*) and $S(4)$ were equivalent and that each was true in the class of spaces having Property C. A space $X$ has Property $C$ if for every separated closed set $B$ there exist disjoint continua $L$ and $M$ in $X$ such that $B \subset(L \cup M)$ and $L \cap B \neq \varnothing \neq M \cap B$. In [4], an example of a uniformly locally connected, connected separable metric space without Property $\mathrm{C}$ was given.

Finally in [3], the author showed that $S(6)$ obtained whenever $X$ was compact and in [1], Harold Bell and the author gave an example of a multicoherent,

Received by the editors October 16, 1978 and, in revised form, October 1, 1979; presented to the Society, January 25, 1979.

AMS (MOS) subject classifications (1970). Primary 54F55. 
1-dimensional, plane Peano continuum for which $S(7)$ fails. Since $S(n+1)$ implies $S(n)$, this meant, that $S(n)$, for $n>6$, was in general false.

The principal objective of this note is to prove that $S(4)$ obtains when we assume that $X$ is a perfectly normal space.

THEOREM. If $X$ is a perfectly normal space then $S(4)$ (or equivalently (*)) holds for $\boldsymbol{X}$.

2. Definitions and preliminary results. A continuous surjection $f: X \rightarrow Y$ is nonalternating if for every $y \in Y$, if $X \backslash f^{-1}(y)=P \cup Q$ is a separation, then $f(P) \cap f(Q)=\varnothing[8$, p. 127].

LEMMA 1 [2, LeMMA 1]. A continuous surjection $f: X \rightarrow[-1,1]$ is nonalternating if and only if for every $s \in(-1,1), X \backslash f^{-1}(s)$ has exactly two components, i.e. $f^{-1}([-1, s))$ and $f^{-1}((s, 1])$ are connected sets.

A continuous surjection $f: X \rightarrow Y$ is interior at $y \in Y$, if whenever $U$ is an open set in $X, U \cap f^{-1}(y) \neq \varnothing$, implies that $y$ is interior to $f(U)$. The set of points in $Y$ at which $f$ is interior is denoted by $g(f)$. Let $\mathscr{D}$ denote the set of dyadic rationals in $(-1,1)$.

LEMma 2. Let $X$ be perfectly normal and let $A$ and $B$ be nonempty disjoint closed subsets of $X$. Then there exists a nonalternating map $f: X \rightarrow[-1,1]$ such that $-1 \in f(A), 1 \in f(B), \mathscr{D} \subseteq \mathscr{G}(f)$ and $f(A \cup B) \cap \mathscr{G}(f)=\varnothing$. Furthermore, if $A$ and $B$ are connected, then $f(A)=-1$ and $f(B)=1$.

Proof. The proof is identical to that of Lemma 4 of [2].

LeMma 3. Let $f: X \rightarrow[-1,1]$ be a continuous surjection and let $-1<s<1$. Then $s \in \mathcal{G}(f)$ if and only if $\mathrm{cl}^{-1}([-1, s)) \cap \mathrm{cl}^{-1}((s, 1])=f^{-1}(s)$. In particular if

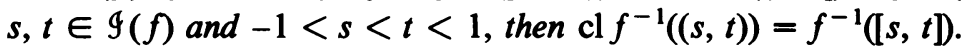

Proof. The continuity of $f$ yields that $\operatorname{cl}^{-1}([-1, s)) \cap \operatorname{cl} f^{-1}\left((s, 1] \subseteq f^{-1}(s)\right.$. Now suppose $s \in G(f)$ and $x \in f^{-1}(s)$ and let $U$ be any connected open set containing $x$. Since $f(U)$ is connected and $s$ is interior to $f(U), f(U)$ meets $[-1, s)$ and $f(U)$ meets $(s, 1]$. This implies that $x \in \operatorname{cl} f^{-1}([-1, s)) \cap \mathrm{cl}^{-1}((s, 1])$ as required. Now suppose $f^{-1}(s) \subseteq \operatorname{cl} f^{-1}([-1, s)) \cap \operatorname{cl} f^{-1}((s, 1])$ and let $x \in f^{-1}(s)$. Then if $U$ is any connected open set containing $x$, there are points $u \in U \cap$ $f^{-1}([-1, s))$ and $v \in U \cap f^{-1}((s, 1])$. Then $s \in(u, v) \subseteq f(U)$ and $f$ is interior at $s$. This completes the proof.

LEMMA 4. Let $X$ be perfectly normal. Then every pair of nonempty disjoint continua in $X$ can be separated by a continuum if and only if for every nonalternating function $f: X \rightarrow[-1,1]$, if $s, t \in G(f),-1<s<t<1$, then $f^{-1}((s, t))$ is connected.

Proof. The sufficiency: Let $A$ and $B$ be nonempty disjoint continua in $X$. By Lemma 2, there exists a nonalternating map $f: X \rightarrow[-1,1]$ such that $f(A)=$ $-1, f(B)=1$ and $D \subseteq \mathscr{G}(f)$. Since $f$ is interior at $-\frac{1}{2}$ and $\frac{1}{2}, \operatorname{cl} f^{-1}\left(\left(-\frac{1}{2}, \frac{1}{2}\right)\right)=f^{-1}\left(\left[\frac{1}{2}, \frac{1}{2}\right]\right)$ and so by our hypothesis $C=f^{-1}\left[-\frac{1}{2}, \frac{1}{2}\right]$ is a continuum in $X$. Clearly $C$ separates $A$ and $B$ in $X$ as required. 
Proof of The Necessity. Let $s<t$ and let $s, t \in \mathscr{T}(f)$. By Lemma $1, f^{-1}([-1, s))$ and $f^{-1}((t, 1])$ are connected sets and since $s, t \in \mathscr{T}(f), \mathrm{cl}^{-1}([-1, s))=$ $f^{-1}([-1, s])$ and $\operatorname{cl} f^{-1}((t, 1])=f^{-1}([t, 1])$ and each of these sets is a continuum. Now, by our hypothesis some continuum $C$ in $f^{-1}((s, t))$ separates $f^{-1}([-1, s])$ and $f^{-1}([t, 1])$ in $X$. Let $K$ be the component of $f^{-1}((s, t))$ that contains $C$. We assert that $f(K)=(s, t)$. To see this, suppose $s \notin \mathrm{cl} f(K)$. Then $K$ is a component of $X \backslash f^{-1}(t)$ and so $X \backslash f^{-1}(t)$ has at least three components, $K$, and the components meeting $f^{-1}(-1)$ and $f^{-1}(1)$ respectively. Of course this contradicts Lemma 1 and so $s \in \operatorname{cl} f(K)$. Similarly $t \in \operatorname{cl} f(K)$ and $f(K)=(s, t)$. Now, this implies $K=$ $f^{-1}(s, t)$. For if $Q$ is any other component of $f^{-1}((s, t))$, it follows, by the argument above, that $f(Q)=(s, t)$. This means $K$ cannot separate $f^{-1}([-1, s])$ and $f^{-1}([t, 1])$ and this is a contradiction. Thus $f^{-1}((s, t))=K$ is connected and this completes the proof.

\section{Proof of main result.}

THEOREM. Let $X$ be a locally connected, connected, perfectly normal space. Then $S(4)$ holds for $X$.

Proof. We will prove the equivalent statement: $(*) \equiv X$ is unicoherent if and only if every pair of nonempty disjoint continua can be separated by a continuum. By Theorem 4.7 of [9, p. 49], if $X$ is unicoherent, every pair of nonempty disjoint continua in $X$ can be separated by a continuum.

Now suppose that every pair of nonempty disjoint continua in $X$ can be separated in $X$ by a continuum, but that $X$ is not unicoherent. Then $X=H \cup K$ where $H$ and $K$ are closed and connected sets and $H \cap K=A \cup B$ where $A$ and $B$ are disjoint, nonempty, closed sets. By Lemma 2, there exists a nonalternating map $f: X \rightarrow[-1,1]$ such that $-1 \in f(A), 1 \in f(B), \mathscr{D} \subseteq \mathscr{G}(f)$ and $\mathscr{G}(f) \cap f(A \cup B)$ $=\varnothing$.

Now $f^{-1}(0)$ separates $f^{-1}(-1) \cap A$ and $f^{-1}(1) \cap B$ and $f^{-1}(0) \cap(A \cup B)=\varnothing$. This implies that $f^{-1}(0)$ is not connected, say $f^{-1}(0)=C \cup D$, where $C$ and $D$ are nonempty disjoint closed subsets of $X$. Since $X$ is perfectly normal, there exists a continuous surjection $g: X \rightarrow[-1,1]$ with $g^{-1}(-1)=C, g^{-1}(1)=D$ and $g^{-1}(0)=$ $f^{-1}(\{-1,1\})$.

By Lemma $4, f^{-1}((-1,1))=\cup_{i=1}^{\infty} f^{-1}\left(-1+2^{-i}, 1-2^{-i}\right)$ is connected. But this is impossible since $f^{-1}((-1,1))=X \backslash g^{-1}(0)=g^{-1}([-1,0)) \cup g^{-1}((0,1])$ is a separated set. Hence $X$ is unicoherent and this completes the proof.

COROLlaRY. Let $X$ be a locally connected, connected, perfectly normal space. Then the following are equivalent:

Property I. If $A$ and $B$ are disjoint closed subsets of $X$, and $x, y \in X$ are such that neither $A$ nor $B$ separates $x$ and $y$ in $X$ then $A \cup B$ does not separate $x$ and $y$ in $X$.

Property I' (Phragmen-Brouwer Property). If neither of the disjoint closed subsets $A$ and $B$ of $X$ separates $X$, then $A \cup B$ does not separate $X$.

Property II (Brouwer Property). If $M$ is a closed, connected subset of $X$ and $C$ is a component of $X \backslash M$, then the boundary of $C$ is a closed and connected set. 
Property III (Unicoherence). If $X=A \cup B$, where $A$ and $B$ are closed and connected, then $A \cap B$ is connected.

Property IV. If $F$ is a closed subset of $X$, and $C_{1}, C_{2}$ are disjoint components of $X \backslash F$ which have the same boundary, $B$, then $B$ is closed and connected.

Property V. If $A$ and $B$ are disjoint closed subsets of $X, a \in A, b \in B$, then there exists a closed, connected subset $C$ of $X \backslash(A \cup B)$ which separates $a$ and $b$.

Property VI. Every pair of disjoint continua in $X$ can be separated by a continuum in $X$.

Property VII. For every nonalternating map $f: X \rightarrow[-1,1]$ and every pair $s, t \in$ $g(f), s<t, f^{-1}((s, t))$ is connected.

Property VIII. If neither of the disjoint closed connected sets $A$ and $B$ of $X$ separates $X$, then $A \cup B$ does not separate $X$.

Proof. The equivalence of I-V is Theorem 4.10 of [9]. III is equivalent to VI by the theorem above and VI is equivalent to VII by Lemma 4. The equivalence of VIII and VI is Theorem 2 of [3].

REMARK. Some of the equivalences above are known to hold under weaker conditions, e.g., Theorem 4.10 of [9] assumes that $X$ is completely normal and Theorem 2 of [3] merely requires that $X$ be normal. In [6], J. H. V. Hunt shows that I, $I^{\prime}$ and III are equivalent without the assumption of any separation axioms.

It is unknown whether $S(5)$ or $S(6)$ obtains for noncompact spaces.

\section{REFERENCES}

1. Harold Bell and R. F. Dickman, Jr., A counterexample to a conjecture of A. H. Stone, Proc. Amer. Math. Soc. 71 (1978), 305-308.

2. R. F. Dickman, Jr., Some mapping characterizations of unicoherence, Fund. Math. 78 (1973), 27-35.

3. __ Multicoherent spaces, Fund. Math. 91 (1976), 219-229.

4. R. F. Dickman, Jr., R. A. McCoy and L. R. Rubin, C-separated sets in certain metric spaces, Proc. Amer. Math. Soc. 40 (1973), 285-290.

5. R. F. Dickman, Jr. and L. R. Rubin, C-separated sets and unicoherence, Fund. Math. 82 (1973), 19-27.

6. J. H. V. Hunt, The Phragmen-Brouwer Theorem for separated sets, Bol. Soc. Sci. Mexicana 19 (1974), 26-35.

7. A. H. Stone, Incidence relations in unicoherent spaces, Trans. Amer. Math. Soc. 65 (1949), 427-447.

8. G. T. Whyburn, Analytic topology, Amer. Math. Soc. Colloq. Publ., vol. 28, Amer. Math. Soc., Providence, R. I., 1942.

9. R. L. Wilder, Topology of manifolds, Amer. Math. Soc. Colloq. Publ., vol. 32, Amer. Math. Soc., Providence, R. I., 1949.

Department of Mathematics, Virginia Polytechnic Institute and State University, BlacksBURG, VIRGINIA 24061 